\title{
Effects of tenuifolin on rest/wake behaviour in zebrafish
}

\author{
ZI-WEN CHEN ${ }^{1}$, CHAO-BAO PENG $^{2}$, ZHONG PEI $^{3}$, MENG-RUO ZHANG $^{1}$, \\ TIAN-CHAN YUN ${ }^{1}$, ZHI-MIN YANG ${ }^{4}$ and FU-PING XU ${ }^{4}$
}

\begin{abstract}
${ }^{1}$ Health Construction Administration Center, The Second Clinical Medicine College, Guangzhou University of Chinese Medicine, Guangzhou, Guangdong 510405; ${ }^{2}$ Department of Traditional Chinese Medicine, Zhaoqing Hospital of Traditional Chinese Medicine, Zhaoqing, Guangdong 526020; ${ }^{3}$ Department of Neurology, National Key Clinical Department and Key Discipline of Neurology, Guangdong Provincial Key Laboratory for Diagnosis and Treatment of Major Neurological Diseases, The First Affiliated Hospital, Sun Yat-Sen University, Guangzhou, Guangdong 510080; ${ }^{4}$ Health Construction Administration Center, Guangdong Provincial Hospital of Chinese Medicine, Guangzhou University of Chinese Medicine, Guangzhou, Guangdong 510120, P.R. China
\end{abstract}

Received March 4, 2019; Accepted December 30, 2019

DOI: $10.3892 /$ etm.2020.8476

\begin{abstract}
Insomnia is a common sleep disorder with a high prevalence and substantial adverse consequences. There is growing interest in identifying novel therapeutics from herbal medicine. Tenuifolin is a major constituent of the well-known anti-insomnia herb Radix Polygala. The present study investigated the neural activity in response to tenuifolin during rest/wake behaviour in zebrafish and identified the potential biological signalling pathways involved. An automatic video tracking system was used to monitor the behavioural response of zebrafish larvae for $24 \mathrm{~h}$ after treatment with tenuifolin. In total, six rest/wake parameters were measured and visualized with a behavioural fingerprint. Time series analysis was conducted by averaging the total rest and waking activity in $10 \mathrm{~min}$ intervals. A correlation analysis was performed between tenuifolin and well-known compounds to analyse the underlying biological signalling pathways. Reverse transcription-quantitative PCR was also performed to detect the effects of tenuifolin on the transcription of interesting genes associated with the signalling pathways that were potentially involved. The present results suggested tenuifolin significantly increased the total rest time during the dark phase, with a slight effect on the waking activity in zebrafish larvae. This behavioural phenotype induced by tenuifolin is similar to that of selective serotonin reuptake inhibitors and gamma-aminobutyric acid (GABA) agonists. Furthermore,
\end{abstract}

Correspondence to: Professor Zhi-Min Yang or Dr Fu-Ping Xu, Health Construction Administration Center, Guangdong Provincial Hospital of Chinese Medicine, Guangzhou University of Chinese Medicine, 111 Dade Road, Guangzhou, Guangdong 510120, P.R. China E-mail: yangyo@vip.tom.com

E-mail: xufuping163@163.com

Key words: sleep, herbal medicine, tenuifolin, zebrafish, behavioural profiles, neural signalling pathways the expression levels of GABA transporter 1 were significantly increased after tenuifolin treatment. No significant difference was determined in other associated genes in untreated control and tenuifolin-treated larvae. The present results suggested that tenuifolin caused sleep-promoting activity in zebrafish and that these effects may be mediated by the serotoninergic systems and the GABAergic systems.

\section{Introduction}

Insomnia is a serious clinical problem, affecting $~ 30 \%$ of the general worldwide population (1). Chronic insomnia causes impairments in cognitive, physical and daytime functions $(1,2)$. People suffering from insomnia are more prone to accidents and psychiatric disorders (1). Though current well-established pharmacotherapy greatly improves insomnia, it is limited by unwanted negative side effects, including cognitive and memory impairment, tolerance, withdrawal and dependency (3). Modern therapeutic research aims to find safer drugs that act on specific targets.

Phenotypic screening in zebrafish combined with classical pharmacology and in vivo models provides a successful strategy for drug discovery (4-6). Zebrafish are a rapidly developing and high-throughput diurnal vertebrate model that exhibits high conservation of the structure and function of the central nervous system (CNS) compared with mice and humans (7). These attractive features make zebrafish well suited to study sleep and neurological disorders. Various behavioural phenotypes in zebrafish have been generated, including sleep, memory, learning, stress and anxiety (8-11). Furthermore, a study performed by Rihel et al (12) assessed the effects of thousands of psychotropic drugs or compounds on sleep parameters in zebrafish, revealing that there is pharmacological conservation in zebrafish and that the zebrafish model provides an appropriate platform for efficient sleep drug screening.

The advantages of herbal medicine or traditional Chinese treatments for the management of insomnia are being increasingly recognized, particularly for their stable curative effects 
and limited side effects $(13,14)$. A variety of herbal medicines are available for insomnia treatment (14). Radix Polygala (the dried root of Polygala tenuifolia Willd) is a common single herb prescribed for patients with insomnia (15). Previous in vivo studies have revealed that the extract of Radix Polygala can improve cognitive deficits and has antipsychotic, antidepressive and anti-inflammatory activities $(16,17)$. Additionally, exposing mice to Radix Polygala extract resulted in the attenuation of cocaine-induced hyperlocomotion and prolonged pentobarbital-induced sleep duration, suggesting that Radix Polygala has a sedative effect (18-20). Tenuifolin is one of the constitutions of Radix Polygala, which has blood-brain barrier permeability and can be quickly distributed in the brain to perform pharmacological actions (21). Cao et al (15) revealed that tenuifolin enhanced the hypnotic effects of pentobarbital by increasing total sleep time and decreasing sleep latency. In wild-type mice, tenuifolin promoted sleep without influencing the power density of a sleep electroencephalogram, and this effect may have been mediated by the activation of the gamma-aminobutyric acid (GABA)ergic systems and/or the inhibition of the noradrenergic systems (15). However, studies on the sleep-promoting effects of tenuifolin are still limited and there is a lack of verification in other animal species. Considering the advantages of zebrafish in sleep research, the present study investigated the neuroactive effects of tenuifolin on sleep behaviour in zebrafish and the neural signalling pathways that are potentially involved, based on well-established behavioural profiles. The present research aims to better understand the biological effects of tenuifolin and its underlying mechanisms, thus facilitating the development of novel therapeutics for insomnia.

\section{Materials and methods}

Animals. Adult zebrafish of the AB strain (obtained from the Laboratory of Neurology, The First Affiliated Hospital, Sun Yat-sen University) were maintained in a 14/10 h light/dark (L/D) cycle at $28.5^{\circ} \mathrm{C}$ with a recirculating water system. Fertilized eggs were collected from the natural spawning of breeding pairs (male and female ratio of 1:2). Embryos and larvae were kept in standard embryo water $(0.05 \mathrm{~g} \mathrm{KCl}, 0.025 \mathrm{~g}$ $\mathrm{NaHCO}_{3}, 3.5 \mathrm{~g} \mathrm{NaCl} 0.1 \mathrm{~g} \mathrm{CaCl}_{2}$ and $0.1 \%$ methylene blue in 11 distilled water) under a consistent temperature of $28.5^{\circ} \mathrm{C}$ and a photoperiod cycle matching that of the adults in an incubator. The embryo water was changed twice a day and the dead or abnormal embryos were discarded. There is a certain mortality rate in the process of natural hatching of fertilized eggs. All the larvae were disposed of according to local regulation (Regulation on the Administration of Experimental Animals; https://www.labagd.com/index.aspx) following the completion of behavioural testing. All experiments were performed following internationally recognized guidelines on animal welfare (2013 AVMA Guidelines for the Euthanasia of Animals) (22), as well as the Guide for the Care and Use of Laboratory Animals. The present study was approved by the Animal Studies Committee of The Guangzhou University of Chinese Medicine.

Chemical treatments. Tenuifolin (Meilun Bio Co., Ltd.; purity, >98\%; $20 \mathrm{mg}$; Fig. 1) and melatonin (Shanghai Aladdin Bio-Chem Technology Co., Ltd.; purity, 98\%;

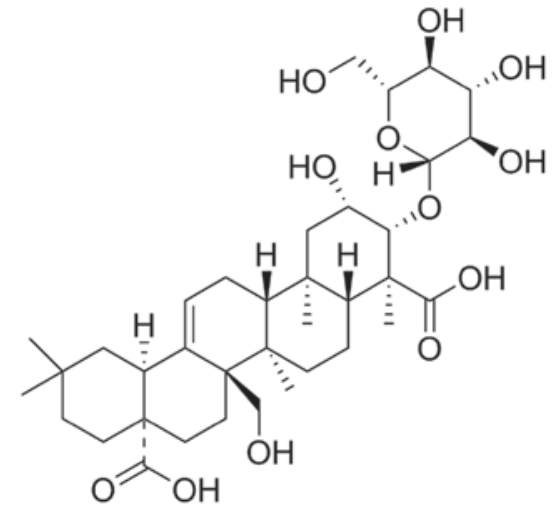

Figure 1. Chemical structure of tenuifolin, $\mathrm{C}_{36} \mathrm{H}_{56} \mathrm{O}_{12}$.

$250 \mathrm{mg}$ ) were dissolved in DMSO to make stock solutions. Larvae at 4 days post-fertilization (dpf) were each transferred to a well of a 96-well plate (one larvae per well) with $300 \mu \mathrm{l}$ standard embryo water. A total of $60 \mu \mathrm{l} 6 \mathrm{X}$ tenuifolin/melatonin solution was added for dilution to the final treatment concentration. Larvae were exposed to drugs beginning at $96 \mathrm{~h}$ post-fertilization (hpf). The following tenuifolin concentrations were tested, 1, 10, 20 and $30 \mu \mathrm{M}$. Standard embryo water served as an untreated control and $10 \mu \mathrm{M}$ melatonin served as a positive control. In addition, the present study used the $\mathrm{GABA}_{\mathrm{A}}$ antagonist, picrotoxin (Shanghai Macklin Biochemical Co., Ltd.; purity, $>98 \%$; $25 \mathrm{mg}$ ). Cotreatment with $30 \mu \mathrm{M}$ tenuifolin and $20 \mu \mathrm{M}$ picrotoxin was also performed. The final DMSO exposure was $<0.3 \%$. All drug concentrations tested in the current study did not cause animal death. Each treatment was tested on 12 larvae and was repeated three times.

Behavioural testing. Behavioural testing was performed for $24 \mathrm{~h}$ beginning at $11 \mathrm{pm}$ at $4 \mathrm{dpf}(110 \mathrm{hpf})$ till $11 \mathrm{pm}$ at $5 \mathrm{dpf}$ using a Viewpoint video tracking system (Viewpoint Life Sciences, Inc.). The 96-well plate was placed into a Zebrabox chamber (Viewpoint Life Sciences, Inc.; http://www.viewpoint.fr) following drug exposure and was immersed in a recirculating water bath that was maintained at $28.5^{\circ} \mathrm{C}(24 \mathrm{~h})$. The experimental parameters were set as follows: Detection threshold, 40; burst, 25; freeze, 4; and bin size, $60 \mathrm{sec}$ (12). All recordings were performed with the same 14/10 h L/D cycle.

Reverse transcription-quantitative PCR (RT-qPCR). RT-qPCR was performed to investigate the effects of tenuifolin on the transcription of genes associated with the signalling pathways that may have been involved. Larvae were exposed to $10 \mu \mathrm{M}$ tenuifolin at $4 \mathrm{dpf}$ for $24 \mathrm{~h}$. They were rapidly paralyzed (200 mg/l MS-222) before sacrifice and RNA extraction. Total RNA was extracted from whole larvae using TRIzol ${ }^{\circledR}$ reagent (Invitrogen; Thermo Fisher Scientific, Inc.) and reverse transcribed into cDNA using a PrimeScript RT reagent kit with gDNA Eraser (Takara Bio, Inc.) in accordance with the manufacturers protocol (reaction conditions were $37^{\circ} \mathrm{C}$ for $15 \mathrm{~min}, 85^{\circ} \mathrm{C}$ for $5 \mathrm{sec} ; 4^{\circ} \mathrm{C}$ ). qPCRs were run on a CFX96 touch instrument (Bio-Rad Laboratories, Inc.) using SYBR Premix Ex Taq II (Takara Bio, Inc). The thermocycling conditions were as follows: $95^{\circ} \mathrm{C}$ 


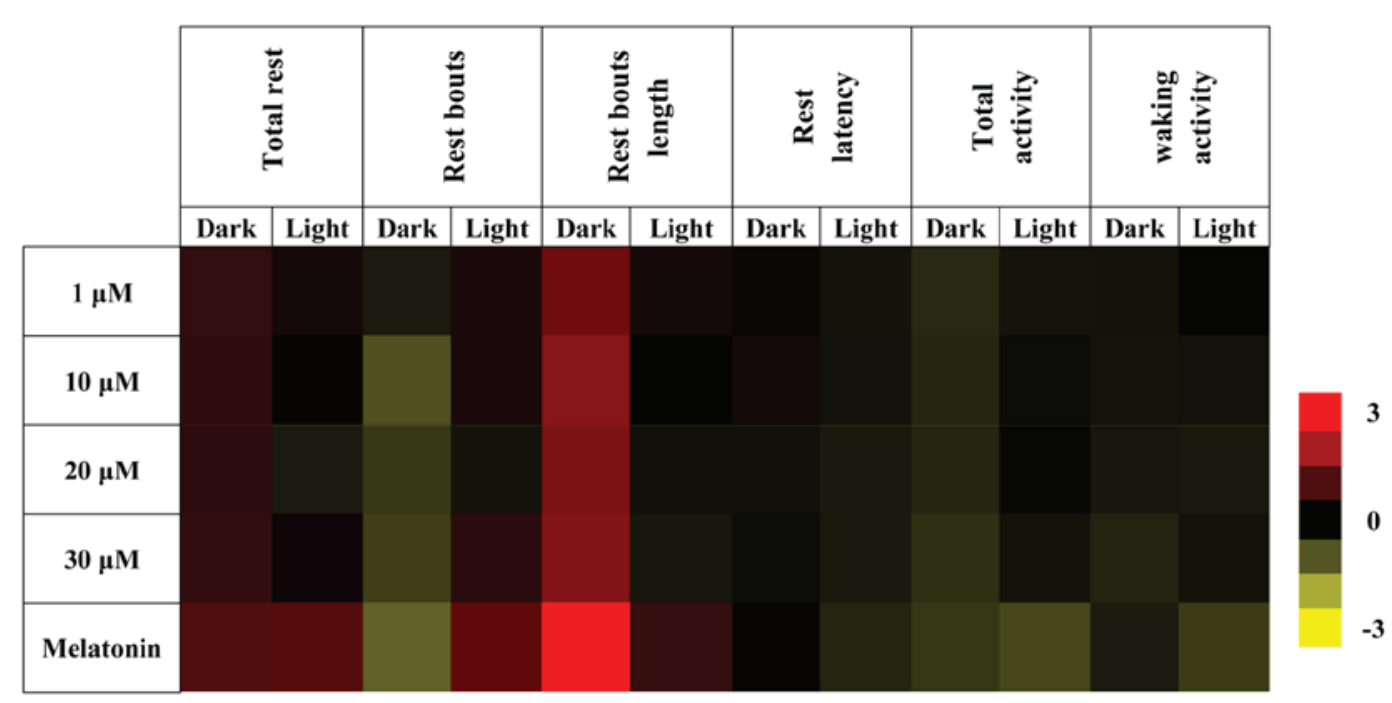

Figure 2. Behavioural fingerprint induced by tenuifolin in zebrafish larvae. Each row represents a treatment and each column represents a behavioural parameter. Each measurement was normalized as standard deviations from the corresponding untreated control and is represented as different colours: Red, > untreated controls; black, untreated controls; yellow, < untreated controls.

for $30 \mathrm{sec}$, followed by 40 cycles of $95^{\circ} \mathrm{C}$ for $5 \mathrm{sec}, 60^{\circ} \mathrm{C}$ for $30 \mathrm{sec}$. The experiment was performed in triplicate with three samples (each sample was pooled from 30 larvae) per group. The sequences of the primers were as follows: Serotonin transporter (sert) protein forward, 5'-CATCTATGCTGA GGCTATTG-3' and reverse, 5'-AAGAATATGATGGCG AAGA-3'; serotonin 1A (5- $\left.\mathrm{HT}_{1 \mathrm{~A}}\right)$ receptor forward, 5'-ATG AGGATGAGCGGGATGTAG-3' and reverse, 5'-CAATCA GCCAGGACCACG-3'; glutamate decarboxylase 1 (gad1) forward, 5'-AACTCAGGCGATTGTTGCAT-3' and reverse, 5'-TGAGGACATTTCCAGCCTTC-3'; GABAA receptor $\alpha 1$ (gabra1) forward, 5'-TCAGGCAGAGCTGGAAGGAT-3' and reverse, 5'-TGCCGTTGTGGAAGAACGT-3'; GABA transporter 1 (gat1) forward, 5'-ATGCTGTTTATCCTGTTCATC CG-3' and reverse, 5'-TGTTGAAGGGGTTGTAGCTCC-3'; and $\beta$-actin ( $\beta$-act) forward, 5'-CATCCATCGTTCACAGGA AGTG-3' and reverse, 5'-TGGTCGTTCGTTTGAATCTCA T-3'. The transcript levels of different genes were normalized to that of $\beta$-act and expressed as the relative expression, which was calculated using the $2^{-\Delta \Delta \mathrm{Cq}}$ method (23).

Data analysis. Data were analysed with R software (version 3.5.0; https://www.r-project.org/). Detectable movement of $<0.1 \mathrm{sec}$ over the course of $1 \mathrm{~min}$ was considered to be $1 \mathrm{~min}$ of rest and a continuous string of rest was considered a 'rest bout'. In total, six rest/wake parameters, including i) total rest (total rest in min); ii) number of rest bouts; iii) rest bout length (average length of rest bouts); iv) rest latency (the length of time between the start of a light transition [on or off] and the appearance of the first rest bout); v) total activity (average time of detected activity in sec, including all rest bouts); and vi) waking activity (total time of detected activity, excluding all 1 min periods of rest), were calculated for day and night. Each parameter was averaged from three repeated experiments and standardized to the matched untreated control to generate a fingerprint. Time series analyses for total rest and waking activity were conducted by averaging these two parameters in 10 min intervals.
Since drugs with shared targets induced similar phenotypes in zebrafish larvae, the comparison of well-known and uncharacterized drugs based on behavioural phenotypes may predict the targets of drugs whose mode of action is poorly understood (12). Distance correlation analysis was therefore performed by calculating Pearson's correlation coefficient to identify known compounds that were similar to tenuifolin in behavioural phenotypes. These compounds were obtained from a well-established behavioural profiling database (12). Each compound was assigned a behavioural fingerprint and in each, different colours indicated the value of different behavioural parameters. By extracting the colour value (red, green and blue), the corresponding behavioural parameters were calculated (24). Among >500 compounds with significantly altered behaviour compared with controls, $\sim 100$ well-characterized compounds were selected for correlation analysis in the present study. Statistical comparisons between treatment groups and untreated group were performed by one-way ANOVA followed by Tukey test. Student's t-test was performed for comparisons between the transcript levels of different genes between the $10 \mu \mathrm{M}$ tenuifolin-treated group and the untreated group. $\mathrm{P}<0.05$ was considered to indicate a statistically significant difference.

\section{Results}

Behavioural fingerprint induced by tenuifolin in zebrafish larvae. Consecutive $24 \mathrm{~h}$ behavioural monitoring revealed that at all tested concentrations, tenuifolin increased total rest and rest bout lengths during the dark phase, and caused a reduction in total activity and waking activity when compared with the untreated control. The light-phase total rest was increased at all tested concentration except for $20 \mu \mathrm{M}$. The light-phase rest bout lengths was increased at low concentrations (1 and $10 \mu \mathrm{M})$, and was decreased in high concentrations (20 and $30 \mu \mathrm{M})$. The number of rest bouts was decreased during the dark phase and increased during the light phase in all tenuifolin-treated group except for $20 \mu \mathrm{M}$ group compared with the untreated control 
A

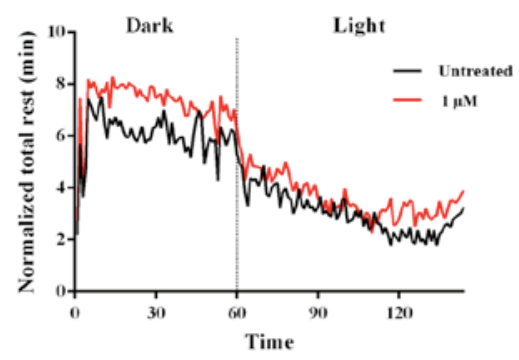

B

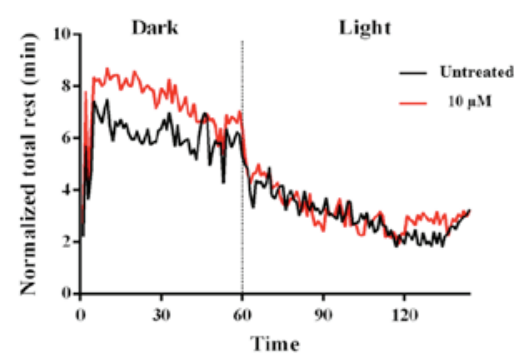

总 C

D
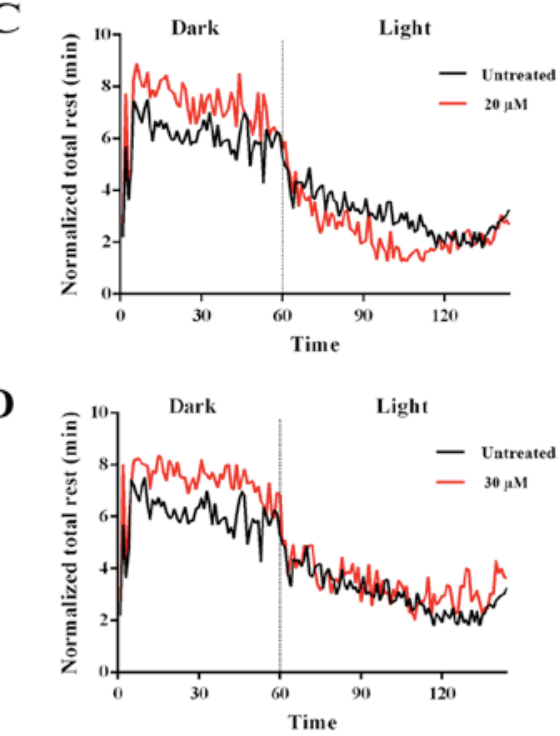

$\mathbf{E}$

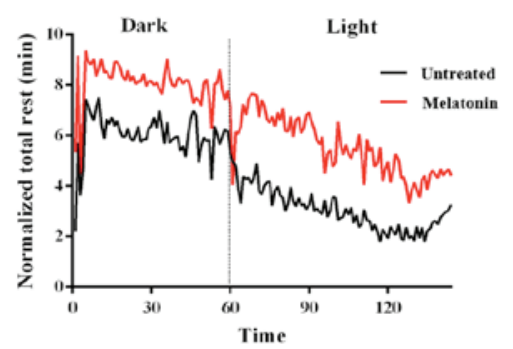

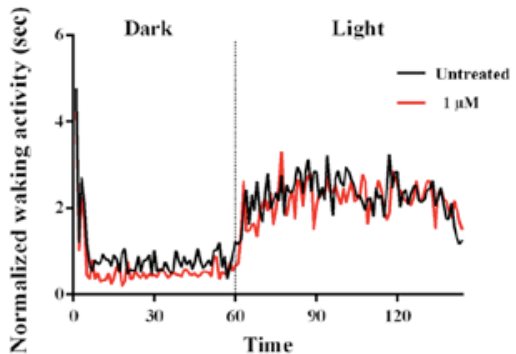
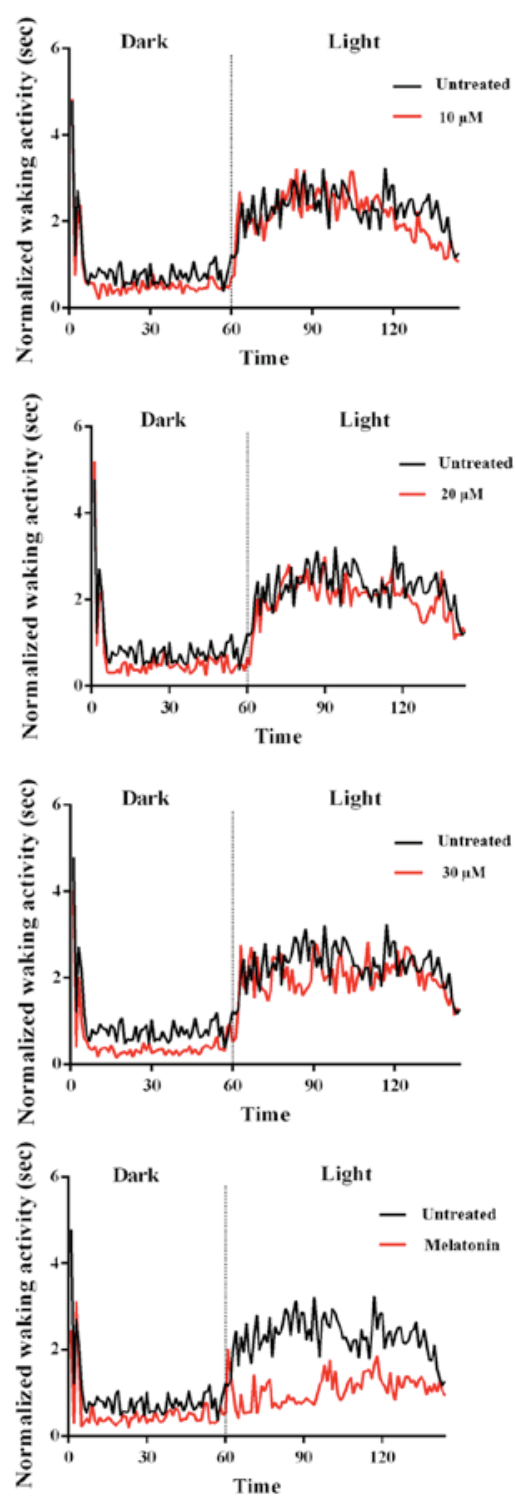

Figure 3. Time series analysis of total rest and waking activity. The average rest and waking activity in 10 min intervals were calculated and normalized to the matched untreated control. (A) The normalized total rest and waking activity of larvae treated with $1 \mu \mathrm{M}$ tenuifolin. The red trace indicates the average of the $1 \mu \mathrm{M}$ tenuifolin group and the black trace indicates the average of the untreated control group. (B) The normalized total rest and waking activity of larvae treated with $10 \mu \mathrm{M}$ tenuifolin. The red trace indicates the average of the $10 \mu \mathrm{M}$ tenuifolin group and the black trace indicates the average of the untreated control group. (C) The normalized total rest and waking activity of larvae treated with $20 \mu \mathrm{M}$ tenuifolin. The red trace indicates the average of the $20 \mu \mathrm{M}$ tenuifolin group and the black trace indicates the average of the untreated control group. (D) The normalized total rest and waking activity of larvae treated with $30 \mu \mathrm{M}$ tenuifolin. The red trace indicates the average of the $30 \mu \mathrm{M}$ tenuifolin group and the black trace indicates the average of the untreated control group. (E) The normalized total rest and waking activity of larvae treated with melatonin. The red trace indicates the average of the melatonin group and the black trace indicates the average of the untreated control group.

group. In addition, rest latency was decreased during the light phase in response to tenuifolin treatment. However, the responses to tenuifolin differed during the dark phase, with low concentrations 1 and $10 \mu \mathrm{M}$ increasing the rest latency and high concentrations 20 and $30 \mu \mathrm{M}$ decreasing the rest latency (Fig. 2).
Time series analysis and quantitative analysis of behavioural parameters. The present study performed time series analysis and quantitative analysis for two principal parameters reflecting the rest/wake behaviours; the total rest and waking activity. The present results suggested that both the untreated control 

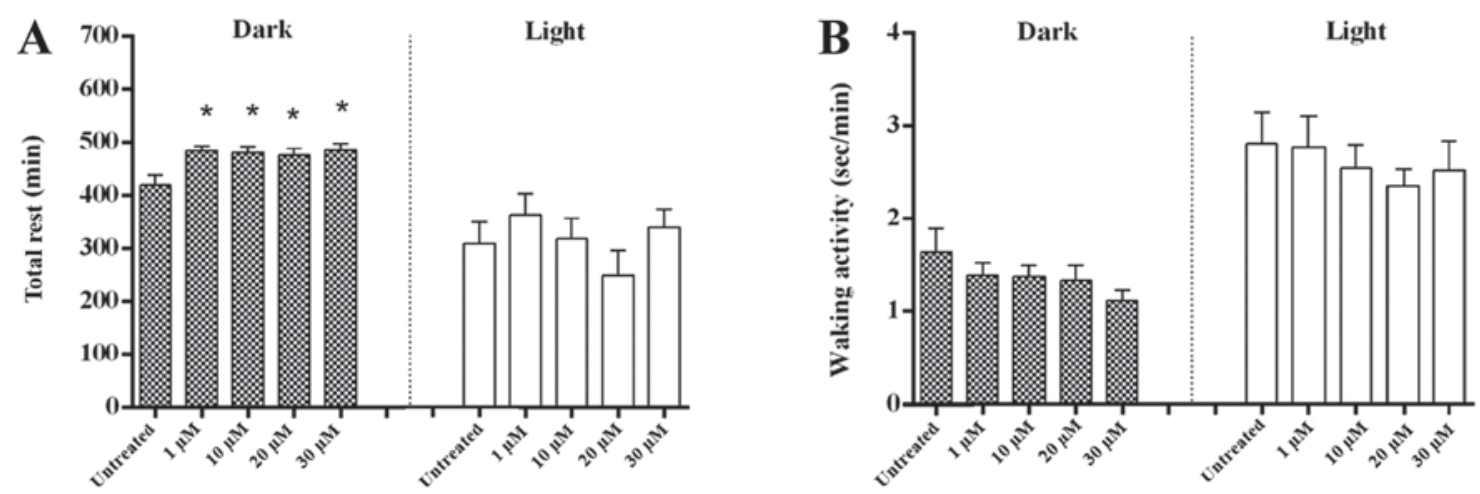

Figure 4. (A-B) Quantitative analysis of total rest and waking activity. Data are presented as mean \pm SEM. "P $<0.05$ vs. the untreated control group. (A) Quantitative analysis of total rest. (B) Quantitative analysis of waking activity.

\begin{tabular}{|c|c|c|c|c|c|}
\hline Target & Compound & $1 \mu \mathrm{M}$ & $10 \mu \mathrm{M}$ & $20 \mu \mathrm{M}$ & $30 \mu \mathrm{M}$ \\
\hline a2-Adrenaline agonist & Clonidine & & & & \\
\hline a2-Adrenaline agonist & Guanabenz Acetate & & & & \\
\hline a1-Adrenaline antagonist & Nicergoline & & & & \\
\hline a1-Adrenaline antagonist & Prazosin & & & & \\
\hline B-Adrenaline antagonist & Carvedilol Tartrate & & & & \\
\hline 5-HT-1A-serotonin agonist & Buspirone & & & & \\
\hline 5-HT-2/3-serotonin agonist & N-Methylquipazine & & & & \\
\hline 5-HT-2/3-serotonin agonist & Quipazine & & & & \\
\hline 5-HT-1A-serotonin antagonist & WAY-100635 Maleate Salt & & & & \\
\hline 5-HT-1A-serotonin antagonist & P-MPPF Dihydrochloride & & & & \\
\hline 5-HT-1A-serotonin antagonist & S(-)-UH-301 Hydrochloride & & & & \\
\hline 5-HT-2/3-serotonin antagonist & Noreyclobenzaprine & & & & \\
\hline 5-HT-reuptake inhibitors & 6-Nitroquipazine & & & & \\
\hline 5-HT-reuptake inhibitors & Fluvoxamine Maleate & & & & \\
\hline 5-HT-reuptake inhibitors & Zimelidine Dihydrochloride & & & & \\
\hline 5-HT-reuptake inhibitors & Paroxetine Hydrochloride & & & & \\
\hline 5-HT-reuptake inhibitors & Fluoxetine Hydrochloride & & & & \\
\hline D1-Dopamine Agonist & (+/-)-Chloro-APB Hydrobromide & & & & \\
\hline D1-Dopamine Agonist & CY 208-243 & & & & \\
\hline D1-Dopamine Agonist & R(+)-6-Bromo-APB Hydrobromide & & & & \\
\hline D1-Dopamine Agonist & SKF 83565 Hydrobromide & & & & \\
\hline D2-Dopamine Agonist & R(-)Apomorphine Hydrochloride Hemihydrate & & & & \\
\hline D2-Dopamine Agonist & Dipropyl-6,7-ADTN & & & & \\
\hline D2-Dopamine Agonist & Pergolide Methanesulfonate & & & & \\
\hline D2/3-Dopamine Agonist & Quinelorane Dihydrochloride & & & & \\
\hline D2/3-Dopamine Agonist & (-)-Quinpirole & & & & \\
\hline D2/3-Dopamine Agonist & Ropinirole Hydrochloride & & & & \\
\hline D1-Dopamine Antagonist & R(-)-SCH-12679 Maleate & & & & \\
\hline D3-Dopamine Antagonist & GR 103691 & & & & \\
\hline GABA-A-GABA Agonist & GBLD-345 & & & & \\
\hline GABA-A-GABA Agonist & Avermectin B1 & & & & \\
\hline HI-no HERG block-Histamine Antagonist & Trimeprazine Tartrate & & & & \\
\hline HI-no HERG block-Histamine Antagonist & Clemizole Hydrochloride & & & & \\
\hline H1-no HERG block-Histamine Antagonist & Chloropyramine Hydrochloride & & & & \\
\hline H1-no HERG block-Histamine Antagonist & Loratadine & & & & \\
\hline Adenosine Agonist & 2-Chloroadenosine & & & & \\
\hline NMDA-Glutamate Antagonist & CNS- 1102 & & & & \\
\hline mGluR5-Glutamate Antagonist & SIB 1757 & & & & \\
\hline
\end{tabular}

Figure 5. Correlation analysis of tenuifolin with known compounds. Each row represents a different compound and its corresponding target, belonging to a different signalling pathway. Each column indicates a different tenuifolin concentration. Rectangles in different colours represent the correlation coefficient of a pair of drugs: Red, high correlation; blue, low correlation. The results with a Pearson correlation coefficient $>0.5$ are presented.

and tenuifolin-treated larvae demonstrated apparent day-night rhythms, with a longer duration of rest during the dark phase and higher activity levels during the light phase (Fig. 3A-D). The total rest time of the dark phase, but not of the light phase, was significantly increased in larvae treated with tenuifolin compared with the untreated control (Fig. 4A; P<0.05). No significant difference in activity levels was demonstrated at any concentration of tenuifolin tested during either the dark or light phase compared with the untreated control (Fig. 4B).
Correlation analysis and target prediction. To identify the neural signalling pathways associated with tenuifolin treatment, Pearson's correlation coefficient between behavioural phenotypes elicited by tenuifolin and by known neuroactive compounds was calculated (Fig. S1). The present results indicated the effects of tenuifolin on larvae were similar to the behavioural changes induced by selective serotonin reuptake inhibitors (SSRIs) and GABA-A-GABA agonists (Fig. 5; Pearson correlation coefficient, $>0.75$ ). 


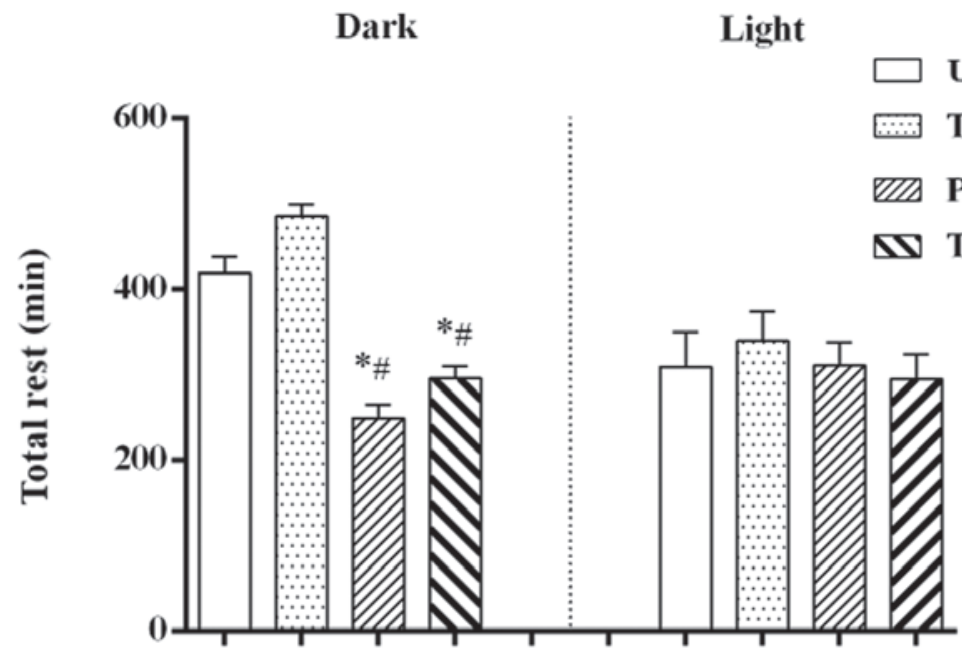

Figure 6. Effects on total rest after cotreatment with tenuifolin and picrotoxin. Data are presented as mean $\pm \mathrm{SEM}$. ${ }^{*} \mathrm{P}<0.05$ vs. the untreated control group. ${ }^{\#} \mathrm{P}<0.05$ vs. $30 \mu \mathrm{M}$ tenuifolin.
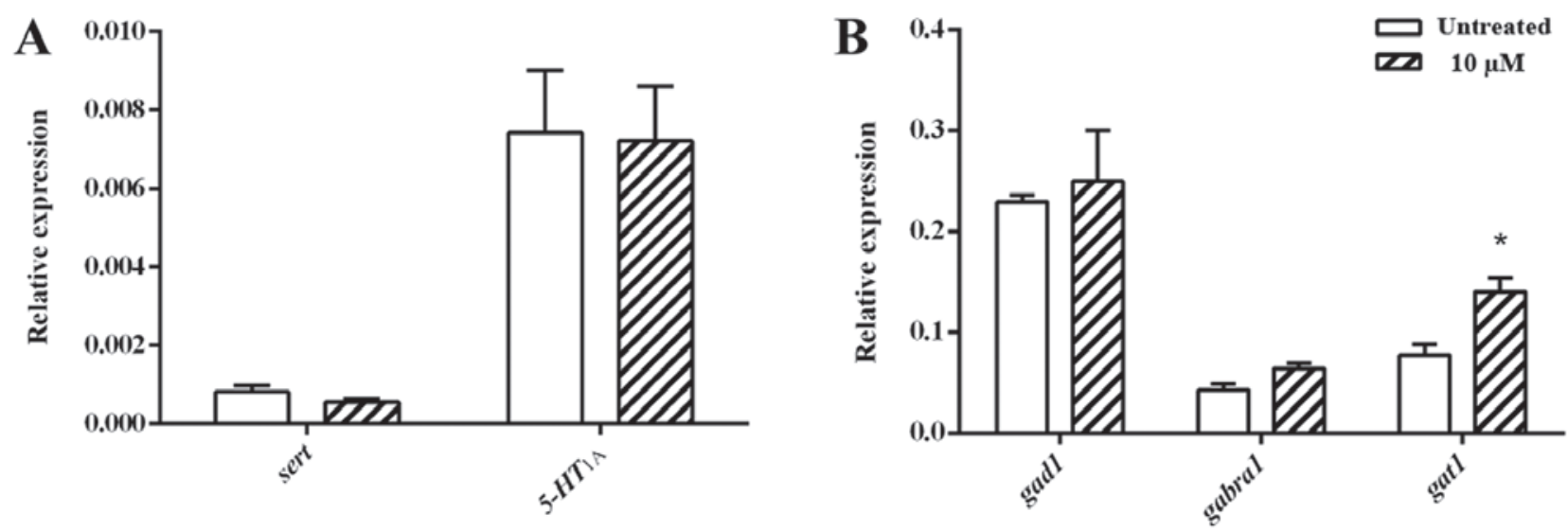

Figure 7. Expression of genes associated with the serotoninergic and GABAergic signalling pathways after tenuifolin treatment. The transcript levels of different genes were normalized to that of $\beta$-act. (A) Expression of genes associated with the serotoninergic signalling pathways after tenuifolin treatment. (B) Expression of genes associated with the GABAergic signalling pathways after tenuifolin treatment. Data are presented as mean \pm SEM. ${ }^{*} \mathrm{P}<0.05$ vs. the untreated control group. $\beta$-act, $\beta$-actin; GABA, gamma-aminobutyric acid; sert, serotonin transporter; 5 - $\mathrm{HT}_{1 \mathrm{~A}}$ serotonin $1 \mathrm{~A}$; gad1, glutamate decarboxylase 1 ; gabra1, GABAA receptor $\alpha 1$; gat1, GABA transporter 1 .

Cotreatment with tenuifolin and picrotoxin. Since the effects induced by tenuifolin may be associated with those of GABA-A-GABA agonists, cotreatment with tenuifolin and the $\mathrm{GABA}_{\mathrm{A}}$ antagonist picrotoxin was performed to determine whether the effects of the antagonist could be counteracted by tenuifolin. The present results identified that $20 \mu \mathrm{M}$ picrotoxin significantly decreased dark-time rest. However, cotreatment with $30 \mu \mathrm{M}$ tenuifolin and picrotoxin for $24 \mathrm{~h}$ slightly counteracted the effect induced by picrotoxin (Fig. 6).

Transcription levels of serotonin- and GABA-associated genes. The transcription levels of genes associated with the serotoninergic and GABAergic signalling pathways were examined after tenuifolin treatment. No significant difference was observed in serotonin-associated genes in the untreated control and tenuifolin-treated larvae (Fig. 7A). The transcription of gad1 and gabra1 was slightly upregulated after tenuifolin treatment compared with the untreated control.
There was a significant increase in gat1 transcript expression (Fig. 7B) in the tenuifolin-treated group compared with the untreated control group.

\section{Discussion}

Zebrafish are important for sleep research and the correlation of drugs with biological targets and rest/wake regulation based on zebrafish behavioural profiling have been well documented $(8,12)$. To the best of our knowledge, the present study was the first to characterize of the effects of tenuifolin on the sleep behaviours of zebrafish and to predict the biological signalling pathways targeted by tenuifolin using quantitative behavioural profiling.

In the present study, tenuifolin was revealed to be a hypnotic constituent of Radix Polygala that promotes sleep with a pattern that is similar to the physiological pattern of mice (15). Consistent with this sleep-promoting effect in mice, the present behavioural monitoring results suggested that 
zebrafish larvae exposed to tenuifolin exhibited significantly increased dark-time rest, while maintaining a normal day-night rhythm, with a slight effect on waking activity. Therefore, tenuifolin may exhibit activity across different species, supporting the biological effects of tenuifolin on sleep regulation. In the present study tenuifolin significantly increased total rest only in the dark phase, with little effect on light phase rest or on waking activity. These selective effects for distinct behavioural parameters and phases suggested that different parameters may be regulated by distinct mechanisms during different phases. In addition, the selective regulation of sleep also suggested that the normal day-night rhythm and daytime activity were unaffected. Therefore, comparing with the current well-established pharmacotherapies to improve sleep, tenuifolin may cause less daytime functional impairments, such as fatigue and increased risk of accidents during work or while driving. In addition, tenuifolin is one of the constitutions from Radix Polygala (21). Radix Polygala has long been used for in vivo study, which have demonstrated that Radix Polygala extract improves cognitive deficits (16). Based on these, it is possible that tenuifolin may exert effects on cognitive processing or memory. However, to understand whether tenuifolin exerts these effects, further study is required. Future study should focus on associated behavioural changes such as memory and cognition, and genetically manipulated zebrafish should be utilized.

The present results suggested that tenuifolin induced a behavioural phenotype resembling that of 5-HT-reuptake inhibitors and GABA agonists and it should be presumed that serotoninergic systems and GABAergic systems may be involved in the underlying mechanisms that contributed to the sleep-promoting effects of tenuifolin. Serotonin has long been known to involved in the regulation of sleep (25). However, its role in sleep regulation remains controversial. It was revealed that the destruction of the raphe nuclei, the areas containing most of the serotoninergic neurons $(\sim 80 \%)$ of the brain, caused complete insomnia (26). However, the serotonin level of raphe nuclei and the firing rate of brain stem motoneurons receiving serotoninergic signalling were higher during the day-time (wake) than during the night-time (sleep) (27). Generally, SSRIs improve insomnia in patients with depression (12). However, in mammals, the effects of SSRIs on sleep have demonstrated biphasic effects, with short-term exposure promoting sleep and long-term exposure inducing the opposite effect (12,28-30). Similar to mammalian phenotypes, complicated phenotypes were observed in zebrafish in response to drugs regulating serotonin (12). These contradictory results revealed the complexity of serotonin in sleep regulation, which may be due to a direct effect. Serotonin could exert indirect effects by modulating other non-serotoninergic systems (25). At present, there remains a lack of studies assessing the correlations between serotoninergic systems and tenuifolin. The aim of the present study was to identify the potential molecular changes associated with serotoninergic systems after tenuifolin exposure and to investigate the expression of sert and $5-H T_{1 \mathrm{~A}}$ receptors. Sert is responsible for transporting serotonin from the synaptic cleft and regulating extracellular serotonin (31). The $5-H T_{1 \mathrm{~A}}$ receptor is a $5-\mathrm{HT}$ receptor. In the present study the expression of these genes did not change after tenuifolin treatment. A similar result was also revealed after exposure to the selective SSRI fluoxetine in a previous study (31). However, instead of using whole larvae, the same experiments were separately performed in two brain regions (rostral and caudal segments) and the spinal cord, which showed a specific fluoxetine-induced a decrease in both genes in the spinal cord, but no change was observed in the two brain regions (31). Accordingly, it could be presumed that tenuifolin exerts its effects by targeting specific regions of the CNS. Future study should focus on different regions of the CNS.

GABAergic neurons are active during sleep, inhibiting activating and arousing systems, thereby promoting the initiation and maintenance of non-rapid eye movement sleep at multiple levels $(32,33)$. The widely used hypnotic medications in clinical settings, benzodiazepines and nonbenzodiazepines, are modulators that target the $\mathrm{GABA}_{\mathrm{A}}$ receptor and act by enhancing the inhibitory effects of GABA (34). In the present study, GABAergic systems may represent a second pathway that tenuifolin affects. To investigate whether the effects of $\mathrm{GABA}_{\mathrm{A}}$ antagonist could be counteracted by tenuifolin, cotreatment with tenuifolin and the $\mathrm{GABA}_{\mathrm{A}}$ antagonist, picrotoxin, was also performed. The present results indicated that treatment with picrotoxin decreased dark-time rest, which was different from the effects of tenuifolin. However, cotreatment of tenuifolin only slightly reversed the effects of picrotoxin during dark-time rest. This may be associated with the limitation of sample size in the present study and therefore future studies should address this. In addition, picrotoxin is one of many $\mathrm{GABA}_{\mathrm{A}}$ antagonists and others should be tested. The ratio of picrotoxin and tenuifolin may also exert effects on results, indicating that multiple ratios of drug doses should be investigated.

Additionally, to correlate the behavioural changes with molecular changes that mediated GABAergic signalling, the expression of gad1, gabra1 and gat1 genes were investigated. Gad1 is a major marker associated with GABA-mediated synaptic function, which catalyses GABA synthesis from glutamate (35-37). Gabral is a subunit of the $\mathrm{GABA}_{\mathrm{A}}$ receptor involved in GABA-mediated postsynaptic signalling (37). Gat1, the most abundant gat in the brain, serves an important role in the regulation of GABA levels in the synaptic cleft (38-40). Tenuifolin induced the expression of gat 1 in the present study, indicating a correlation between increased transcript levels of gat1 and the sleep-promoting effects of tenuifolin on zebrafish larvae. A previous study by Cao et al (15) determined that tenuifolin significantly increased GABA levels in the ventrolateral preoptic area, locus coeruleus and perifornical area of mice. Thus, the present results supported the association of GABAergic systems with the hypnotic activity of tenuifolin at the molecular level. However, there are certain limitations to the present study, as many downstream gene products participate in the regulation of the assessed signalling pathway. Further studies investigating multiple molecular levels and using genetically manipulated zebrafish deficient in genes associated with the GABAergic systems are therefore required. In addition, the present study did not perform a comparison of dark or light environments. A future study will aim to detect the alternations in the gene expression patterns along the L/D cycle after tenuifolin exposure.

Overall, the present results indicated that tenuifolin treatment promoted dark-time sleep and elicited a behavioural phenotype that is correlated with that of SSRIs and GABA 
agonists. Changes in the gene expression of associated genes further supported the involvement of GABAergic systems. In the current study, a novel strategy based on zebrafish behavioural profiling was applied to identify the potential biological targets of tenuifolin. The present results identified that tenuifolin exhibited promise for treating insomnia.

\section{Acknowledgements}

Not applicable.

\section{Funding}

The present study was supported by grants from the National Natural Science Foundation of China (grant nos. 81974560 and 81503515), Natural Science Foundation of Guangdong Province (grant no. 2015A030310255), Guangdong Provincial Key Laboratory for Diagnosis and Treatment of Major Neurological Diseases (grant no. 2017B030314103) and The Southern China International Cooperation Base for Early Intervention and Functional Rehabilitation of Neurological Diseases (grant no. 2015B050501003).

\section{Availability of data and materials}

The data generated during the present study are included in this published article.

\section{Authors' contributions}

ZMY and FPX conceived and designed the experiments. ZWC, MRZ and TCY performed the experiments. ZWC, CBP and ZP analysed the data. ZWC drafted the manuscript. ZMY and FPX revised the manuscript. All authors have read and given final approval of the manuscript before submission.

\section{Ethics approval and consent to participate}

All experiments were performed in accordance with the Guide for the care and use of laboratory animals and approved by the Animal Studies Committee of the Guangzhou University of Chinese Medicine.

\section{Patient consent for publication}

Not applicable.

\section{Competing interests}

The authors declare that they have no competing interests.

\section{References}

1. Roth T: Insomnia: Definition, prevalence, etiology, and consequences. J Clin Sleep Med 3 (Suppl 5): S7-S10, 2007.

2. Léger D, Morin CM, Uchiyama M, Hakimi Z, Cure S and Walsh JK: Chronic insomnia, quality-of-life, and utility scores: Comparison with good sleepers in a cross-sectional international survey. Sleep Med 13: 43-51, 2012.

3. Asnis GM, Thomas M and Henderson MA: Pharmacotherapy treatment options for insomnia: A primer for clinicians. Int J Mol Sci 17: E50, 2015.
4. Swinney DC: The contribution of mechanistic understanding to phenotypic screening for first-in-class medicines. J Biomol Screen 18: 1186-1192, 2013.

5. Ek F, Malo M, Åberg Andersson M, Wedding C, Kronborg J, Svensson P, Waters S, Petersson P and Olsson R: Behavioral analysis of dopaminergic activation in zebrafish and rats reveals similar phenotypes. ACS Chem Neurosci 7: 633-646, 2016.

6. Ellis LD and Soanes KH: A larval zebrafish model of bipolar disorder as a screening platform for neuro-therapeutics. Behav Brain Res 233: 450-457, 2012.

7. Levitas-Djerbi T and Appelbaum L: Modeling sleep and neuropsychiatric disorders in zebrafish. Curr Opin Neurobiol 44: 89-93, 2017.

8. Zhdanova IV, Wang SY, Leclair OU and Danilova NP: Melatonin promotes sleep-like state in zebrafish. Brain Res 903: 263-268, 2001.

9. Blank M, Guerim LD, Cordeiro RF and Vianna MR: A one-trial inhibitory avoidance task to zebrafish: Rapid acquisition of an NMDA-dependent long-term memory. Neurobiol Learn Mem 92: 529-534, 2009.

10. Pather S and Gerlai R: Shuttle box learning in zebrafish (Danio rerio). Behav Brain Res 196: 323-327, 2009.

11. Egan RJ, Bergner CL, Hart PC, Cachat JM, Canavello PR, Elegante MF, Elkhayat SI, Bartels BK, Tien AK, Tien DH, et al: Understanding behavioral and physiological phenotypes of stress and anxiety in zebrafish. Behav Brain Res 205: 38-44, 2009.

12. Rihel J, Prober DA, Arvanites A, Lam K, Zimmerman S, Jang S, Haggarty SJ, Kokel D, Rubin LL, Peterson RT and Schier AF: Zebrafish behavioral profiling links drugs to biological targets and rest/wake regulation. Science 327: 348-351, 2010.

13. Sánchez-Ortuño MM, Bélanger L, Ivers H, LeBlanc M and Morin CM: The use of natural products for sleep: A common practice? Sleep Med 10: 982-987, 2009.

14. Wing YK: Herbal treatment of insomnia. Hong Kong Med J 7: 392-402, 2001

15. Cao Q, Jiang Y, Cui SY, Tu PF, Chen YM, Ma XL, Cui XY, Huang YL, Ding H, Song JZ, et al: Tenuifolin, a saponin derived from Radix Polygalae, exhibits sleep-enhancing effects in mice. Phytomedicine 23: 1797-1805, 2016.

16. Ling Y, Li Z, Chen M, Sun Z, Fan M and Huang C: Analysis and detection of the chemical constituents of Radix Polygalae and their metabolites in rats after oral administration by ultra high-performance liquid chromatography coupled with electrospray ionization quadrupole time-of-flight tandem mass spectrometry. J Pharm Biomed Anal 85: 1-13, 2013.

17. Cheng MC, Li CY, Ko HC, Ko FN, Lin YL and Wu TS: Antidepressant principles of the roots of Polygala tenuifolia. J Nat Prod 69: 1305-1309, 2006.

18. Shin EJ, Oh KW, Kim KW, Kwon YS, Jhoo JH, Jhoo WK, Cha JY, Lim YK, Kim IS and Kim HC: Attenuation of cocaine-induced conditioned place preference by Polygala tenuifolia root extract. Life Sci 75: 2751-2764, 2004.

19. Yao Y, Jia M, Wu JG, Zhang H, Sun LN, Chen WS and Rahman K: Anxiolytic and sedative-hypnotic activities of polygalasaponins from Polygala tenuifolia in mice. Pharm Biol 48: 801-807, 2010.

20. Lee CI, Han JY, Hong JT and Oh KW: 3,4,5-Trimethoxycinnamic acid (TMCA), one of the constituents of Polygalae Radix enhances pentobarbital-induced sleeping behaviors via GABAAergic systems in mice. Arch Pharm Res 36: 1244-1251, 2013.

21. Ma B, Li X, Li J, Zhang Q, Liu Y, Yang X, Sun J, Yao D, Liu L, Liu X and Ying H: Quantitative analysis of tenuifolin concentrations in rat plasma and tissue using LC-MS/MS: application to pharmacokinetic and tissue distribution study. J Pharm Biomed Anal 88: 191-200, 2014.

22. Wallace CK, Bright LA, Marx JO, Andersen RP, Mullins MC and Carty AJ: Effectiveness of rapid cooling as a method of euthanasia for young zebrafish (Danio rerio). J Am Assoc Lab Anim Sci 57: 58-63, 2018.

23. Livak KJ and Schmittgen TD: Analysis of relative gene expression data using real-time quantitative PCR and the 2(-Delta Delta C(T)) method. Methods 25: 402-408, 2001.

24. Wang YN, Hou YY, Sun MZ, Zhang CY, Bai G, Zhao X and Feng XZ: Behavioural screening of zebrafish using neuroactive traditional Chinese medicine prescriptions and biological targets. Sci Rep 4: 5311, 2014.

25. Melancon MO, Lorrain D and Dionne IJ: Exercise and sleep in aging: Emphasis on serotonin. Pathol Biol (Paris) 62: 276-283, 2014.

26. Dugovic C: Role of serotonin in sleep mechanisms. Rev Neurol (Paris) 157: S16-S19, 2001. 
27. Heym J, Steinfels GF and Jacobs BL: Activity of serotonin-containing neurons in the nucleus raphe pallidus of freely moving cats. Brain Res 251: 259-276, 1982

28. Pastel RH and Fernstrom JD: Short-term effects of fluoxetine and trifluoromethylphenylpiperazine on electroencephalographic sleep in the rat. Brain Res 436: 92-102, 1987.

29. Sommerfelt L and Ursin R: Behavioral, sleep-waking and EEG power spectral effects following the two specific 5-HT uptake inhibitors zimeldine and alaproclate in cats. Behav Brain Res 45: 105-115, 1991.

30. Weber M, Talmon S, Schulze I, Boeddinghaus C, Gross G, Schoemaker $\mathrm{H}$ and Wicke KM: Running wheel activity is sensitive to acute treatment with selective inhibitors for either serotonin or norepinephrine reuptake. Psychopharmacology (Berl) 203: 753-762, 2009.

31. Airhart MJ, Lee DH, Wilson TD, Miller BE, Miller MN and Skalko RG: Movement disorders and neurochemical changes in zebrafish larvae after bath exposure to fluoxetine (PROZAC). Neurotoxicol Teratol 29: 652-664, 2007.

32. Jones BE: Neurobiology of waking and sleeping. Handb Clin Neurol 98: 131-149, 2011.

33. Holst SC and Landolt HP: Sleep-Wake Neurochemistry. Sleep Med Clin 13: 137-146, 2018.

34. Richey SM and Krystal AD: Pharmacological advances in the treatment of insomnia. Curr Pharm Des 17: 1471-1475, 2011.

35. Martin DL and Rimvall K: Regulation of gamma-aminobutyric acid synthesis in the brain. J Neurochem 60: 395-407, 1993.
36. Soghomonian JJ and Martin DL: Two isoforms of glutamate decarboxylase: Why? Trends Pharmacol Sci 19: 500-505, 1998.

37. Hortopan GA, Dinday MT and Baraban SC: Spontaneous seizures and altered gene expression in GABA signaling pathways in a mind bomb mutant zebrafish. The J Neurosci 30: 13718-13728, 2010.

38. Kang TC, Kim HS, Seo MO, Park SK, Kwon HY, Kang JH and Won MH: The changes in the expressions of gamma-aminobutyric acid transporters in the gerbil hippocampal complex following spontaneous seizure. Neurosci Lett 310: 29-32, 2001.

39. Meldrum BS and Rogawski MA: Molecular targets for antiepileptic drug development. Neurotherapeutics 4: 18-61, 2007.

40. Chen NH, Reith ME and Quick MW: Synaptic uptake and beyond: The sodium- and chloride-dependent neurotransmitter transporter family SLC6. Pflugers Arch 447: 519-531, 2004.

This work is licensed under a Creative Commons Attribution-NonCommercial-NoDerivatives 4.0 International (CC BY-NC-ND 4.0) License. 